\title{
AVALAÇÃO DO ESTADO NUTRICIONAL DE PACIENTES INTERNADOS EM UM HOSPITAL UNIVERSITÁRIO
}

\section{EVALUATION OF NUTRTIONAL STATUS OF HOSPIAUZED PATIENTS FROM AN UNIVERSITY HOSPITAL}

\author{
Poliana Coelho CABRAL ${ }^{1}$ \\ Maria Goretti Pessoa de Araújo BURGOS² \\ Alcinda de Queiroz MEDEIROS 3 \\ Ana Karina Teixeira TENÓRIO ${ }^{3}$ \\ Charla Cavalcante FEITOZA ${ }^{3}$
}

\section{RESUMO}

O objetivo deste trabalho foi avaliar o estado nutricional de pacientes internados no Hospital das Clínicas da Universidade Federal de Pernambuco, na faixa etária de 15 a 89 anos. A amostra escolhida ao acaso, foi composta por 154 indivíduos de ambos os sexos (74 homens e 80 mulheres). Durante o estudo foram coletados os dados antropométricos, os hábitos alimentares e as causas do internamento. As análises mostraram uma grande instabilidade nutricional, com elevadas prevalências tanto de baixo peso como de excesso de peso. Com relação aos hábitos alimentares foi observada pobreza e monotonia nos cardápios. Dos pacientes entrevistados, a maioria mencionou apenas seis tipos de alimentos comuns como sendo seus favoritos (arroz e feijão, carne bovina, galinha, macarrão e pão). Por outro lado, esse estudo também deixou claro a alta freqüência de tabus alimentares, o que pode limitar o consumo de vários nutrientes. Em termos de admissões ás clínicas médica e cirúrgica, a proporção para ambos o grupos foi muito similar.

Termos de indexação: avaliação nutricional, pacientes internados, hábitos alimentares, tabu alimentar.

\begin{abstract}
The purpose of this research was to evaluate the nutritional status of hospitalized patients, aged 15 to 89 years old, from the Hospital das Clínicas - Universidade Federal de Pernambuco - Brazil. The sample, chosen at random, consisted of 154 individuals of both sexes (74 males and 80 females). Anthropometric data, food habits and reasons for hospitalization were recorded during the assessment. The analysis showed a great nutritional instability, with high prevalences of underweight and overweight. Regarding food habits, it was observed a poor and monotonous menu, resulted from a lack of variety.
\end{abstract}

(1) Mestre em Nutrição e Saúde Pública, Nutricionista Clínica do Hospital das Clínicas da Universidade Federal de Pernambuco.

(2) Mestre em Nutrição Experimental, Nutricionista Clínica do Hospital das Clínicas da Universidade Federal de Pernambuco.

(3) Nutricionistas, Residentes do Hospital das Clínicas da Universidade Federal de Pernambuco. 
Of the patients interviewed, the majority mentioned only six kinds of common foods as their favourites (rice and beans, beef, chicken, macaroni and bread). On the other hand, this study also made clear the high frequency of food taboos, which might limit the consumption of various nutrients. In terms of admissions to the medical and surgical services, the proportion for both groups was very similar.

Index terms: nutritional assessment, impatients, food habits, food taboo

\section{INTRO DUÇÃO}

Nosúltimos anos têm crescido mundialmente o interesse pelo estado nutricional do paciente hospitalizado, uma vez que já é de conhecimento geral os efeitos da desnutrição sobre a morbimortalidade desse grupo em particular.

Segundo BLACKBURN \& THORNTON (1979), a inanição pura e simples só acarretará altas taxas de mortalidade quando ocorrer perda de $40 \%$ do peso, enquanto que a associação da desnutrição com enfermidade já pode levar a morte quando houver perda ponderal de $25 \%$.

Infelizmente, a desnutrição hospitalar é extremamente freqüente entre os indivíduos adultos internados. Em alguns estudos já foi encontrada prevalência tão elevada quanto 50\% (BISTRIAN et al., 1974; BISTRIAN et al., 1976; WEINSIER et al., 1979).

Apesar da realidade mostrada acima, em nosso meio as informações ainda são bastante escassas. Desse modo, levando-se em consideração que, compete ao profissional de nutrição dimensionar a situação, intervindo quando necessário, e tendo consciência também da precária literatura envolvendo nossos pacientes, o objetivo desse trabalho foi o de descrever e analisar a situação nutricional dos indivíduos adultos internados no Hospital das Clínicas da Universidade Federal de Pernambuco (UFPE).

\section{MATERIAL E MÉTODOS}

\section{Amostra gem}

Esse estudo de caráter transversal foi realizado com os pacientes internados no Hospital das Clínicas, da Universidade Federal de Pernambuco (UFPE), no período de julho a agosto de 1996.

A amostra foi selecionada através de amostragem aleatória e sistemática, onde de posse da relação das enfermarias, por andar e ala do hospital, procedia-se a escolha da primeira, pulando-se sempre uma a partir dela. Adotando tal critério, a amostra foi composta inicialmente por 154 indivíduos de ambos os sexos com idade acima de 15 anos, o que representa cerca de $48 \%$ do total de pacientes internados, tendo como base um número de leitos igual a 400 e uma taxa de ocupação de $80 \%$.

Durante a fase de coleta dos dados antropométricos, ficou evidenciado que, para essa parte do estudo em particular, havia a impossibilidade de utilização de 21 componentes da amostra, em virtude dos seguintes obstáculos: 13 pacientes não deambulavam; 6 pacientes apresentavam edema significativo, 2 pacientes tinham menos de 18 anos.

Nas duas primeiras situações, ou havia a impossibilidade de aferição das medidas, ou não se podia confiar no peso registrado. Já no último caso, como só foi encontrado dois pacientes ainda na adolescência, eles foram eliminados dessa fase da análise, com o objetivo de tornar o grupo ainda mais homogêneo. Desse modo, para o estudo antropométrico, a amostra ficou em torno de 133 pacientes (42\%) do total de internamentos.

\section{Avaliação do estado nutricional}

Para avaliar o estado nutricional do grupo em estudo foi utilizado o Índice de Massa Corporal (IMC) definido pela fórmula

$$
\mathrm{IMC}=\frac{\text { Peso }(\mathrm{Kg})}{\text { Altura }\left(\mathrm{m}^{2}\right)}
$$

Este indicador vem sendo largamente recomendado na análise da situação nutricional de adultos (GARROW, 1983; ANJOS, 1992; ANSELMO, et al., 1992; CABRAL, 1994). A classificação utilizada foi a proposta por GARROW 
(1983) e adotada pela Organização Mundial de Saúde (COITINHO et al., 1991). que é a seguinte:

$$
\begin{array}{ll}
\text { IMC }<20,0 & \text { Baixo Peso } \\
\text { IMC } \geq 20,0 \text { e }<25,0 & \text { Normal } \\
\text { IMC } \geq 25,0 \text { e }<30,0 & \text { Sobrepeso } \\
\text { IMC } \geq 30,0 & \text { Obesidade }
\end{array}
$$

Em caráter complementar, com o objetivo de reforçar aos resultados, também foram incluídos a prega cutânea do tríceps (PCT), a circunferência do braço (CB) e a circunferência muscular do braço (CMB), cuja classificação seguiu os critérios estabelecidos por FRISANCHO (1981).

Durante a etapa deelaboração do estudo, também se pensou na utilização dos parâmetros bioquímicos (proteína total, albumina, hemoglobina, hematócrito, linfócitos totais) entretanto, devido a pouca representatividade do conjunto desses dados nos prontuários dos pacientes, essa análise tornou-se inviável.

\section{Hábitose ta bus a limenta res}

Além dos dados antropométricos foram levantadas também (no momento da entrevista com o paciente) informações sobre os hábitos alimentares. Ressaltando-se os alimentos preferidos, os de menor aceitação e aqueles que por algum motivo eram rejeitados durante a doença e o internamento (tabus alimentares). Esse procedimento foi baseado na livre resposta, ou seja, foi solicitado ao paciente que respondesse as seguintes perguntas:

1. Quais os alimentos que você mais gosta?

2. Quais os alimentos que você não gosta?

3. Existe algum alimento que você não come quando está doente?

Se for sim, qual?

\section{Doenças ma is freqüentes}

Para a verificação das doenças mais freqüentes (como causa básica do internamento) nesta unidade hospitalar, foi realizado levantamento nos prontuários de todos os pacientes da amostra. Ressalta-se que a maternidade e a pediatria ficaram fora desta pesquisa, tendo em vista que, por razões fisiológicas fogem da homogeneidade do grupo em estudo.

\section{Coleta dos dados}

Os dados foram coletados no período de julho a agosto de 1996, utilizando-se formulário próprio, o qual incluía a identificação do paciente, o diagnóstico principal, os dados antropométricos e bioquímicos e as questões sobre os hábitos e tabus alimentares.

Antes da entrevista, esse formulário foi preenchido com as informações contidas no prontuário. Os instrumentos utilizados na coleta dos parâmetros antropométricos foram: para $150 \mathrm{~kg}$

- Balança da marca Filizola com capacidade

- Antropômetro da balança Filizola com capacidade para $1,90 \mathrm{~m}$

- Paquímetro Harpenden Skinfold calipers, em centímetros com precisão de 2/10milímetros

- Fita métrica de fibra de vidro, em centímetros, com precisão de milímetros.

\section{RESULTADOS E DISC USSÃO}

\section{Distribuição etá ria}

Neste estudo, a idade em anos, verificada no momento da entrevista foi agrupada em oito categorias com intervalo médio de nove anos (Tabela 1).

Observa-se que, quando se estratifica por sexo, 57,6\% das mulheres internadas têm menos de 40 anos, no caso dos homens esse percentual cai para 36,5\%. Estes resultados são semelhantes aos achados por VERAS et al. (1987), no trabalho sobre o crescimento da população idosa no Brasil. Esses autores sugeriram que além dos fatores biológicos, a maior expectativa de vida da mulher devia-se, também, a sua maior preocupação com a saúde, a qual a fazia procurar bem mais cedo a assistência médica. Vale a pena relembrar que a maternidade (setor constituído basicamente por mulheres jovens) não entrou na nossa amostra, portanto essa não seria uma possível variável de confusão no estudo.

Analisando a situação em termos de médias (Tabela 2), constata-se realmente que entre os homens internados a faixa etária é bem superior, 47,5 \pm 10,3 anos contra 37,2 $\pm 15,1$ anos entre as mulheres. 
Tabela 1. Distribuição (\%) de adultos internados por sexo e faixa etária. Hospital das Clínicas/UFPE, Julho/Agosto de 1996.

\begin{tabular}{|c|c|c|c|c|c|c|c|c|c|}
\hline \multirow{2}{*}{$\begin{array}{l}\text { Faixa Etária } \\
\text { (anos) }\end{array}$} & \multicolumn{3}{|c|}{ Homen } & \multicolumn{3}{|c|}{ Mulher } & \multicolumn{3}{|c|}{ Total } \\
\hline & $\mathrm{n}$ & $\%$ & \%Acum & $\mathrm{n}$ & $\%$ & \%Acum & $\mathrm{n}$ & $\%$ & \%Acum \\
\hline $15-19$ & 5 & 6,8 & 6,8 & 8 & 10,0 & 100,0 & 13 & 8,4 & 8,4 \\
\hline $20-29$ & 16 & 21,6 & 28,4 & 26 & 32,6 & 42,6 & 42 & 27,3 & 35,7 \\
\hline $30-39$ & 6 & 8,1 & 36,5 & 12 & 15,0 & 57,6 & 18 & 11,7 & 47,4 \\
\hline $40-49$ & 14 & 18,9 & 55,4 & 17 & 21,2 & 78,8 & 31 & 20,1 & 67,5 \\
\hline $50-59$ & 9 & 12,2 & 67,6 & 9 & 11,2 & 90,0 & 18 & 11,7 & 79,2 \\
\hline $60-69$ & 11 & 14,9 & 82,5 & 8 & 10,0 & 100,0 & 19 & 12,3 & 91,5 \\
\hline $70-79$ & 9 & 12,2 & 94,7 & 0 & - & - & 9 & 5,9 & 97,4 \\
\hline $80-89$ & 4 & 5,3 & 100,0 & 0 & - & - & 4 & 2,6 & 100,0 \\
\hline Total & 74 & 100,0 & - & 80 & 100,0 & - & 154 & 100,0 & - \\
\hline
\end{tabular}

Acum = acumulada.

Tabela 2. Análise de dados antropométricos de adultos internados de ambos os sexos. Hospital das Clínicas/UFPE, Julho/Agosto de 1996.

\begin{tabular}{|c|c|c|c|c|c|c|}
\hline \multirow{2}{*}{ Variáveis } & \multicolumn{3}{|c|}{ Homens } & \multicolumn{3}{|c|}{ Mulheres } \\
\hline & $\bar{X}$ & & $\mathrm{DP}$ & $\bar{X}$ & & DP \\
\hline Idade (anos) & 47,50 & \pm & 10,3 & 37,20 & \pm & 15,10 \\
\hline Altura (m) & 1,64 & \pm & 0,07 & 1,55 & \pm & 0,10 \\
\hline Peso (kg) & 61,00 & \pm & 13,0 & 55,40 & \pm & 12,90 \\
\hline $\operatorname{IMC}\left(\mathrm{Kg} / \mathrm{m}^{2}\right)$ & 22,08 & \pm & 5,31 & 22,64 & \pm & 4,42 \\
\hline PCT (mm) & 10,50 & \pm & 6,20 & 21,20 & \pm & 9,40 \\
\hline $\mathrm{CB}(\mathrm{cm})$ & 30,20 & \pm & 5,90 & 28,30 & \pm & 6,00 \\
\hline $\mathrm{CMB}(\mathrm{cm})$ & 26,20 & \pm & 3,40 & 21,80 & \pm & 3,70 \\
\hline
\end{tabular}

\section{Esta do nutricional}

De acordo com os pontos de corte recomendados pela Organização Mundial de Saúde (OMS), classificação de GARROW (1983), 31,9\% dos homens e $25,0 \%$ das mulheres neste estudo apresentam baixo peso, evidenciando uma situação nutricional deficitária (Tabela 3 ). O resultado acima não é nenhuma surpresa, tendo em vista que reproduz os achados de inúmeros autores (BOLLET \& OWENS, 1973; BISTRIAN et al. 1974; BISTRIAN et al., 1976; BLACKBURN et al., 1979; WEINSIER et al., 1979; WAITZBERG, 1990), que levantaram o problema da desnutrição hospitalar.

Todavia, os dados também são indicativos de particularidades interessantes, em virtude de que os valores encontrados para o excesso de peso de uma forma geral (sobrepeso + obesidade) são similares aos encontrados por COITINHO (1989) para a população nordestina como um todo. Ou seja, estamos diante de um quadro nutricional bastante instável, com elevada prevalência tanto de baixo peso como de sobrepeso e obesidade.

Sabe-se que a capacidade do indivíduo em reagir ao processo patológico depende em grande parte do seu estado nutricional, desse modo, todo o esforço deve ser empregado no sentido de identificar esses pacientes com déficit, com o objetivo de corrigí-lo.

Entretanto, deve-se levar em conta também, que o excesso de peso é um importante fator de risco para um grande número de doenças, tais como hipertensão, dislipidemia, diabetes, certos tipos de câncer, e que essa condição já é um grave problema de saúde pública, afetandonão sóas regiões desenvolvidas bem como as subdesenvolvidas (BJÖRNTORP, 1985; HARRISON, 1985; BRAY, 1991).

Analisando a situação em termos de médias (Tabela 2), constata-se que entre os homens e mulheres internados, os valores exceto para a altura, se situaram dentro da faixa de normalidade, com a prega cutânea do tríceps (PCT), circunferência do braço (CB) e circunferência muscular do braço (CMB) em torno do percentíl 50 do padrão de FRISANCHO (1981), ou seja próximo a mediana dos adultos americanos.

Por outro lado, em relação a altura foi encontrado um grande déficit, o que está de acordo com os dados da Pesquisa Nacional sobre Saúde e Nutrição(INSTITUTO..., 1990) no qual ficou constatado que o nordestino aos 18 anos tinha um déficit em torno de $11 \mathrm{~cm}$ em relação ao padrão americano. 
Tabela 3. Distribuição (\%) de adultos internados de ambos os sexos, segundo os pontos de corte do índice de Massa Corporal (IMC), Hospital das Clínicas - UFPE, Julho/ Agosto - 1996.

\begin{tabular}{lcccccc}
\hline \multirow{2}{*}{ IMC } & \multicolumn{2}{c}{ Homens } & & \multicolumn{2}{c}{ Mulheres } \\
\cline { 2 - 3 } \cline { 6 - 7 } & $\mathrm{n}$ & $\%$ & & $\mathrm{n}$ & $\%$ \\
\hline Baixo Peso $(<20,0)$ & 22 & 31,9 & & 16 & 25,0 \\
Normal $(\geq 20,0<25,0)$ & 31 & 44,9 & & 29 & 45,3 \\
Sobrepeso $(\geq 25,0<30,0)$ & 10 & 14,5 & & 12 & 18,8 \\
Obesidade $(\geq 30,0)$ & 6 & 8,7 & & 7 & 10,9 \\
\hline Total & 69 & 100,0 & & 64 & 100,0 \\
\hline
\end{tabular}

O que pode ser sugerido de acordo com os dados apresentados, é que neste estudo a desnutrição foi muito além dos limites hospitalares, com uma boa parcela da amostra apresentando indícios de um passado nutricional adverso, o que pode ser evidenciado pela baixa estatura. Entretanto muitos desses indivíduos no presente, podem ter acesso a uma alimentação rica em calorias, o que poderia em parte justificar o problema do excesso de peso.

Na Tabela 3, pode-se observar que, apenas $44,9 \%$ dos homens e 45,3\% das mulheres podem ser enquadrados na condição de normalidade. O restante, ou é desnutrido, ou sofre com o problema do excesso depeso. No caso do sexo feminino, quadro semelhante foi encontrado por CABRAL (1994) no seu trabalho com casais nordestinos, no qual $60,8 \%$ e $46,5 \%$ dos homens e mulheres, respectivamente, eram normais e esse grande diferencial entre os sexos ocorreu principalmente em virtude da maior prevalência de excesso de peso entre as mulheres.

\section{Hábitos e tabus alimentares}

No trabalho intitulado "Padrões e hábitos alimentares da população brasileira”, ARRUDA (1981) conclui que a dieta do brasileiro difere basicamente em termos de quantidade e não de qualidade, ou seja, mais por motivos de ordem econômica que cultural.

De acordo com LIMA (1985), 30\% da população urbana e $60 \%$ da população rural do Nordeste, não recebe uma renda que permita a aquisição de uma alimentação adequada. Isto implica (segundo o autor) que a dieta do nordestino é pobre e monótona, constituída apenas de seis itens: pão, café, açúcar, feijão, farinha e arroz.

Em concordância com o exposto acima, essa investigação evidenciou, de forma indireta, a precariedade alimentar do grupo em estudo, tendo em vista que, os alimentos citados como preferidos quase que reproduzem o já escasso cardápio nordestino, ou seja por ordem decrescente de citação temos: arroz com feijão, carne de boi, galinha, macarrão e pão (Figura 1).

Por outro lado, quando o questionamento foi sobre os alimentos de que não se gosta (Figura 2), a maioria dos entrevistados (68\%), estranhamente não referiu nenhum alimento e foi muito comum a ocorrência de respostas do tipo: "Pobre não tem luxo" ou "Foi comida, gosto de tudo".

Outro dado que merece ser analisado é o que se refere aos alimentos rejeitados durante a ocorrência das doenças. É curioso observar a elevada freqüência de respostas nesse item, 92\% dos entrevistados, com uma média de citação de 2 alimentos cada um (Figura 3 ), e entre os alimentos mais citados temos a carne de porco, o abacaxi, as aves de um modo geral e os crustáceos, alimentos tidos como "reimosos" pelos pacientes. Esse resultado está de acordo com os achados de BURGOS (1993) no seu estudo sobre a ação linfagoga dos alimentos.

\section{Doenças freqüentes}

O Hospital das Clínicas da UFPE por se tratar de um hospital escola, apresenta pacientes com uma grande variedade de doenças. Estratificando a amostra, por internamento nas áreas de Clínica Médica e Cirúrgica (Tabela 4), pode-se observar que houve uma certa similaridade em relação ao número de admissões nesses dois serviços (51,9\% e 48,1\% respectivamente). Subdividindo ainda mais, ficou evidenciado que nenhum tipo de doença teve grande destaque, tendo em vista que as maiores prevalências nunca foram superiores a 10,0\%.

Entretanto, convém ressaltar, que essa observação é válida apenas para as doenças como causa básica do internamento (dado que foi coletado nesse estudo) as enfermidades associadas, como por exemplo diabetes e hipertensão, apresentaram elevada freqüência, o que não foi registrado. 


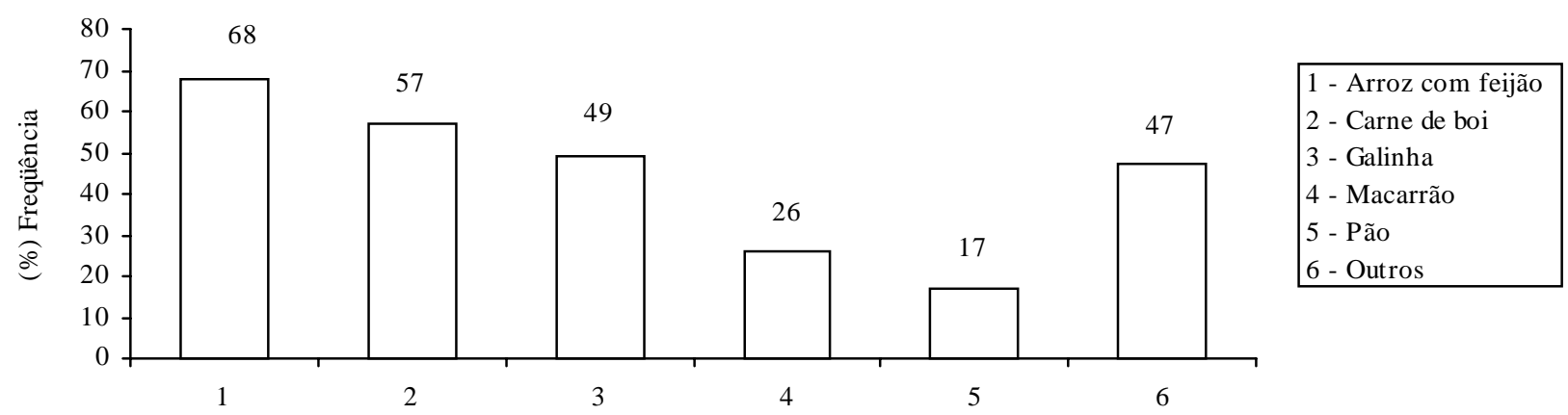

${ }^{(*)}$ Os 154 pacientes pesquisados citaram em média 3 alimentos como seus preferidos

Figura 1. Freqüência de citação* de alimentos tidos como preferidos. Hospital das Clínicas - UFPE Julho/Agosto - 1996.

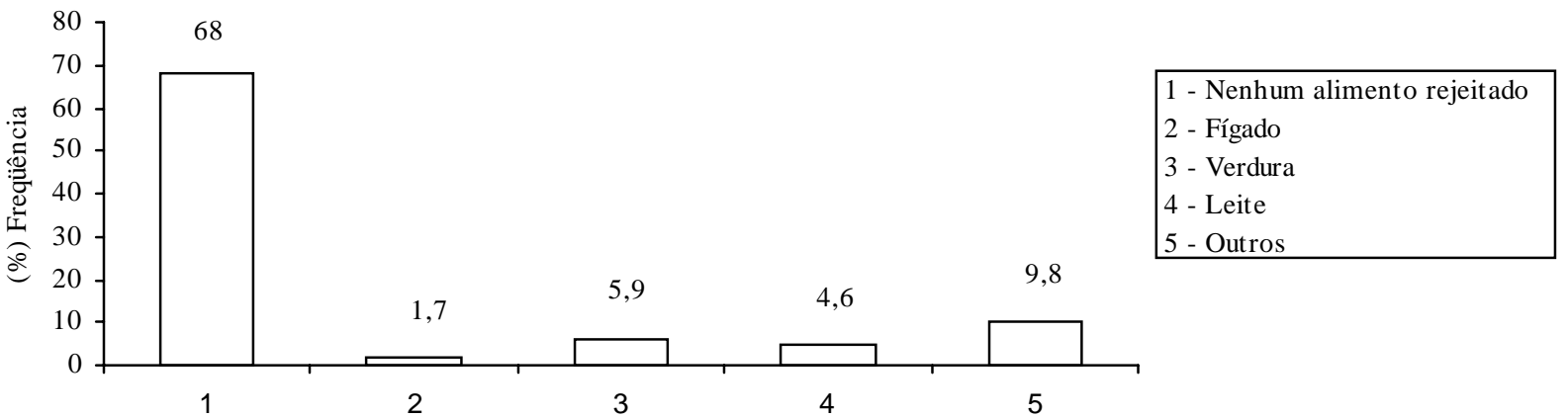

(*) Dos pacientes com resposta positiva nesse ítem (49 dos 154 indivíduos), a média de citação foi de 1 alimento.

Figura 2. Freqüência de citação* de alimentos rejeitados em qualquer situação, Hospital das Clínicas - UFPE, Julho/Agosto - 1996.

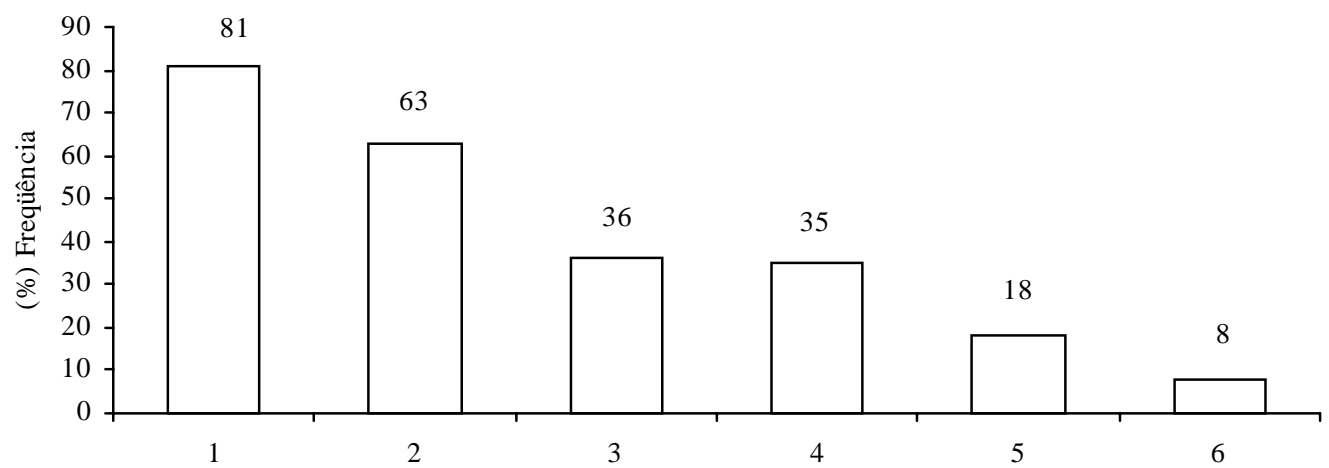

(*) A média de citação foi de 2 alimentos.

Figura 3. Freqüência de citação* de alimentos rejeitados durante a ocorrência de doenças, Hospital das Clínicas - UFPE, Julho/Agosto - 1996. 
Tabela 4. Principais causas de internamento por sexo. Hospital das Clínicas - UFPE, Julho/Agosto - 1996.

\begin{tabular}{|c|c|c|c|c|c|c|}
\hline \multirow{2}{*}{ Causa Básica do Internamento } & \multicolumn{2}{|c|}{ Homens } & \multicolumn{2}{|c|}{ Mulheres } & \multicolumn{2}{|c|}{ Total } \\
\hline & $\mathrm{n}$ & $\%$ & $\mathrm{n}$ & $\%$ & $\mathrm{n}$ & $\%$ \\
\hline \multicolumn{7}{|l|}{ Clínica Médica } \\
\hline IRC em hemodiálise & 6 & 8,2 & 5 & 6,2 & 11 & 7,1 \\
\hline ICC & 3 & 4,0 & 2 & 2,5 & 5 & 3,3 \\
\hline Diabetes descompensada & 2 & 2,7 & 1 & 1,2 & 3 & 1,9 \\
\hline Linfoma & 2 & 2,7 & 2 & 2,5 & 4 & 2,6 \\
\hline Cirrose & 4 & 5,4 & 2 & 2,5 & 6 & 3,9 \\
\hline Câncer de Pulmão & 3 & 4,0 & 4 & 5,0 & 7 & 4,5 \\
\hline DPOC & 3 & 4,0 & 2 & 2,5 & 5 & 3,3 \\
\hline Tuberculose Pulmonar & 4 & 5,4 & 5 & 6,2 & 9 & 5,8 \\
\hline SIDA & 7 & 9,5 & 3 & 6,8 & 10 & 6,5 \\
\hline Outros & 9 & 12,2 & 11 & 13,8 & 20 & 13,0 \\
\hline Sub-Total & 43 & 58,1 & 37 & 46,2 & 80 & 51,9 \\
\hline \multicolumn{7}{|l|}{ Clínica Cirúrgica } \\
\hline Colecistectomia & 4 & 5,4 & 5 & 6,2 & 9 & 5,8 \\
\hline Apendicectomia & 3 & 4,0 & 3 & 3,8 & 6 & 3,9 \\
\hline Gastrectomia & 3 & 4,0 & 2 & 2,5 & 5 & 3,3 \\
\hline Hemorroidectomia & 4 & 5,4 & 2 & 2,5 & 6 & 3,9 \\
\hline Herniorrafia & 4 & 5,4 & 6 & 7,5 & 10 & 6,5 \\
\hline Laparotomia & 2 & 2,7 & 3 & 3,8 & 5 & 3,3 \\
\hline Prostatectomia & 3 & 4,0 & - & - & 3 & 1,9 \\
\hline Cirurgia de Catarata & 3 & 4,0 & 4 & 5,0 & 7 & 4,5 \\
\hline Perineoplastia & - & - & 6 & 7,5 & 6 & 3,9 \\
\hline Histerectomia & - & - & 5 & 6,2 & 5 & 3,3 \\
\hline Outros & 5 & 6,9 & 7 & 8,8 & 12 & 7,8 \\
\hline Sub-Total & 31 & 41,9 & 43 & 53,8 & 74 & 48,1 \\
\hline Total Geral & 74 & 100,0 & 80 & 100,0 & 154 & 100,0 \\
\hline
\end{tabular}

BOLLET \& OWENS (1973), analisando o estado nutricional de certos grupos de pacientes hospitalizados, não encontraram diferença significativa entre os indivíduos pertencentes as clínicas médica e cirúrgica. Neste estudo, no entanto, uma grande parte das cirurgias foram de pequenas intervenções eletivas (herniorrafia, perineoplastia, hemorroidectomia, catarata e outros), enquanto que as admissões na Clínica Médica apresentaram uma elevada prevalência de doenças crônicas consumptivas; síndrome de deficiência imunológica adquirida(SIDA), doença pulmonar obstrutiva crônica (DPOC), insuficiência renal crônica (IRC), cânceres e outros. Desse modo é de se esperar que o estado nutricional nesse segundo grupo apresente um déficit bem superior, o que não foi avaliado nesse trabalho.

\section{CONCLUSÃO}

A desnutrição de um modo geral, interfere de forma muito negativa sobre a capacidade de um indivíduo em reagir contra um processo patológico. Por outro lado, o excesso de peso, também é uma condição de risco que precisa ser corrigida.

Desse modo, a grande instabilidade nutricional, encontrada entre os pacientes adultos internados, com elevada prevalência tanto de baixo 
peso como de sobrepeso e obesidade, pode ser tomada como uma situação que exige um acompanhamento nutricional rigoroso, que deve ser realizado desde admissão até a orientação para alta, pois só assim o profissional de nutrição estará atuando de forma efetiva no processo de recuperação e/ou cura.

\section{REFERÊNCIAS BIBLIOGRÁFICAS}

ANJOS, L.A. Índice de massa corporal (massa corporal - estatura) como indicador do estado nutricional de adultos: revisão da literatura. Revista de Saúde Pública, São, Paulo, v.26, n.6, p.431-436, 1992.

ANSELMO, M.A.C., BURINI, R.C., ANGELELI, A.Y.O., MOTA, N.G.S., CAMPANA, A. O. Avaliação do estado nutricional de indivíduos adultos sadios de classe média. Ingestão energética e protéica, antropometria, exames bioquímicos do sangue e testes de imunocompetência. Revista de Saúde Pública, São Paulo, v.26, n.1, p.46-53, 1992.

ARRUDA, B.K.G. de. Padrões e hábitos alimentares da população brasileira. Brasília : INAN, 1981.67p.

BISTRIAN, B.R., BLACKBURN, G.L., HALLOWEL, E., HADELLE, R. Protein status of general surgical patients. Journal of the American Medical Association, Chicago, v.230, p.858-860, 1974.

BISTRIAN, B.R., BLACKBURN, G.L., VITALE, J., COCHRAN, D., NAYLOR, J. Prevalence of malnutrition in general medical patients. Journal of the American Medical Association, Chicago, v.235, p.1567-1570, 1976.

BJÖRNTORP, P. Regional patterns of fat distribution. Annals of Internal Medicine, Lancaster, v.103, n.6 (part2),p.994-995, 1985.

BLACKBURN, G.L., THORNTON, P.A. Nutritional assessment of the hospitalized patient. Medical Clinic of North America, New York, v.63, p.1103-1115,1979.

BOLLET, A.J., OWENS, S. Evaluation of nutritional status of selected hospitalized patients. American Journal of Clinical Nutrition, Bethesda, v.26, p.931-938,1973.

BRAY, G.A. Obesidad. In: CONOCIMIENTOS actuales sobre nutrición. 6.ed. Washington DC: OPS, 1991. p.28-47.
BURGOS, M.G.P.A. Ação linfagoga de alimentos: fato ou mito ? Recife, 1993. 116p. Dissertação (Mestrado em Nutrição) - Centro de Ciências da Saúde, Universidade Federal de Pernambuco, 1993.

CABRAL, P.C. Homem, mulher e estado nutricional: um estudo em casais do Nordeste brasileiro. Recife, 1994. 143p. Dissertação (Mestrado em Nutrição - Centro de Ciências da Saúde, Universidade Federal de Pernambuco, 1994.

COITINHO, D.C., LEÃO, M.M., RECINE, E., SICHIERI, R. Condições nutricionais da população brasileira: adultos e idosos. Brasília: INAN, 1991.74p.

FRISANCHO, A.R. New norms of upper limb fat and muscle areas for assessment of nutritional status. American Journal of Clinical Nutrition, Bethesda, v.34,n.11,p.2540-2545, 1981.

GARROW, J.S. Indices of adiposity. Nutrition Abstracts and Reviews - Series A, v.53, n.8, p.697-708, 1983.

HARRISON, G.G. Height: weight tables. Annals of Internal Medicine, Lancaster, v.103, n.6 (part2), p.989-994,1985.

INSTITUTO NACIONAL DE ALIMENTAÇÃO E NUTRIÇÃO. Pesquisa Nacional sobre Saúde e Nutrição : perfil de crescimento da população brasileira de 0 a 25 anos. Brasília, 1990. 59p.

LIMA, M.S.S. Estudo sócio-econômico cultural dos hábitos alimentares da Região Nordeste. In: SIMPÓSIO BRASILEIRO DE ALIMENTAÇÃOE NUTRIÇÃO, 7., Niterói, 1984. Anais..., 1984. Rio de Janeiro : Interciência, 1985. p.77-84.

VERAS, R.P., RAMOS, L.R., KALACHE, A. Crescimento da população idosa no Brasil: transformações e conseqüências na sociedade. Revista de Saúde Pública, São Paulo, v.21, n.3, p.225-233, 1987.

WAITZBERG, D.L. Desnutrição: nutrição enteral e parenteral na prática clínica. Rio de Janeiro : Atheneu, 1990.p.143-149.

WEINSIER, R.L., HUNKER, E.M., KRUMDIEK, C.L., BUTTERWORTH, C.E. Hospital malnutrition: a prospective evaluation of general medical patients during the course of hospitalization. American Journal of Clinical Nutrition, Bethesda, v.32, p.418-422, 1979.

YOUNG, G.A. Assessment of protein-calorie malnutrition in surgical patients from plasma proteins and anthropometric measurements. American Journal of Clinical Nutricion, Bethesda, v.31, p.429-435, 1978.

Recebido para publicação em 1 de abril eaceito em 11 denovembro de 1997. 